\title{
E. Edmund Kim, Hyung-Jun Im, Dong Soo Lee, Keon Wook Kang: Atlas and Anatomy of PET/MRI, PET/CT and SPECT/CT
}

\author{
Springer International Publishing, Switzerland, 2016, ISBN: 978-3-319-28650-1
}

\author{
Giuseppe Danilo Di Stasio $^{1} \cdot$ Luigi Mansi ${ }^{2}$
}

Received: 22 September 2017 / Accepted: 9 October 2017 / Published online: 2 November 2017

(C) Springer-Verlag GmbH Germany 2017

This Atlas is edited by Edmund Kim, Professor of Radiologic Sciences at the University of California at Irvine in collaboration with Hyung-Jun Im, Dong Soo Lee and Keon Wook Kang, working at the Nuclear Medicine Department of the Seoul National University in the Republic of Korea.

This volume is a natural evolution of a previous book "Sectional Anatomy: PET/CT and SPECT/CT" by Kim et al. published in 2007. During the last 10 years, we have witnessed a tremendous evolution in hybrid imaging in clinical practice as a result of its ability to provide simultaneous evaluation of morphological and functional information in both physiological and pathological processes leading to a better understanding of the body. In addition, thanks to the constant improvement in instruments, including software for attenuation correction, the integration of PET/MRI images and of new highquality PET/CT and SPECT/CT images has become possible, leading to an increase in both sensitivity and specificity of these techniques.

The book is structured into three sections, one for each hybrid modality, which total 594 pages with more than 100 clinical cases. The first part is dedicated to PET/MRI and is subdivided into paragraphs based on body regions, i.e. brain, head and neck, chest, abdomen, pelvis and musculoskeletal

Luigi Mansi

mansi.luigi@libero.it

1 Centro Interuniversitario per lo Sviluppo Sostenibile (CIRPS), Roma-Naples, Italy

2 Medicina Nucleare, Università Federico II, Naples, Italy system. The core of the book focuses on sectional anatomy of $\mathrm{PET} / \mathrm{CT}$ in oncological cases and is structured differently, the main criterion for organization being the imaging agent itself, either fluorodeoxyglucose (FDG) or other agents. The part on agents other than FDG discusses many different radiopharmaceuticals, including ${ }^{11} \mathrm{C}$-acetate, ${ }^{11} \mathrm{C}$-methionine, ${ }^{11} \mathrm{C}$-PIB, ${ }^{18}$ F-FP-CIT, ${ }^{18}$ F-flumazenil, ${ }^{68} \mathrm{Ga}-\mathrm{RGD}$ and ${ }^{68} \mathrm{Ga}$-DOTATOC. Finally, the last section of the book is devoted to an atlas and the anatomy of SPECT/CT, and includes a thorough discussion of the principle indications. The discussion includes clinical cases of neuroendocrine tumours, bone lesions, including trauma, degenerative disease and avascular necrosis, and a miscellaneous section covering among other subjects gastrointestinal bleeding, abscesses, lymph nodes, accessory spleen. In each chapter there are several clinical cases described briefly but adequately, that introduce the subject, thus leaving the focus on images, functional, morphological or fused. Clear arrows indicate every anatomical structure of relevance, thus guiding the reader to the proper interpretation.

In summary, this Atlas provides a complete view of sectional anatomy of PET/MRI, PET/CT and SPECT/CT that benefits from high-resolution images, balanced by a considerable number of cases described briefly but adequately, and supported by relevant references. The book should be especially helpful to nuclear medicine and radiology residents, who may use it as a great source of ready-to-use knowledge in everyday clinical practice. It may also be useful to both undergraduate and postgraduate students, who will appreciate its importance as a good resource to improve their skill in interpreting hybrid nuclear medicine images. Of course it may also be very valuable in all imaging departments with hybrid machines in helping to improve the interpretation of individual cases in routine practice. 